\title{
ON ANTI-COMMUTATIVE SEMIRINGS
}

\author{
J.S. RATTI and Y.F. LIN \\ Department of Mathematics \\ University of South Florida \\ Tampa, Florida 33620
}

(Received March 28, 1988)

\begin{abstract}
An anticommutative semiring is completely characterized by the types of multiplications that are permitted. It is shown that a semiring is anticommutative if and only if it is a product of two semirings $R_{1}$ and $R_{2}$ such that $R_{1}$ is left multiplicative and $R_{2}$ is right multiplicative.
\end{abstract}

KEY WORDS AND PHRASES. Semiring, anticommutative, isomorphism. 1980 AMS SUBJECT CLASSIFICATION CODES. 16A78

A semiring is a non-empty set $R$ equipped with two binary operations, called addition + and multiplication (denoted by juxtaposition), such that $R$ is multiplicatively a semigroup. additively a commutative semigroup and multiplication is distributive across the addition both from the left and the right.

A semiring $R$ is called anti-commutative if and only if for arbitrary $x, y \in R$ the relation $x \neq y$ always implies $x y \neq y x$.

Let $R_{1}$ and $R_{2}$ be semirings, then $R_{1} \times R_{2}$ is the semiring with the following operations:

$$
\begin{aligned}
& \left(x_{1}, x_{2}\right)+\left(y_{1}, y_{2}\right)=\left(x_{1}+y_{1}, x_{2}+y_{2}\right) \\
& \left(x_{1}, x_{2}\right) \cdot\left(y_{1}, y_{2}\right)=\left(x_{1} y_{1}, x_{2} y_{2}\right) .
\end{aligned}
$$


Suppose $R$ is a commutative semigroup under + , and if we define multiplication in $R$ of type

$$
\begin{array}{ll}
\left(\mathrm{T}_{1}\right) & \mathrm{xy}=\mathrm{x} \text { for all } \mathrm{x}, \mathrm{y} \in \mathrm{R} \\
\text { or } & \\
\left(\mathrm{T}_{2}\right) & \mathrm{xy}=\mathrm{y} \text { for all } \mathrm{x}, \mathrm{y} \in \mathrm{R},
\end{array}
$$

then it is easily seen that $R$ is an anti-commutative semiring.

$A$ natural question that arises is the following: Suppose $R$ is an anti-commutative semiring. Does the multiplication in $R$ have to be of type $\left(T_{1}\right)$ or $\left(T_{2}\right)$ ? to answer this question, we prove the following:

THEOREM 1. A semiring $R$ is anti-commutative if and only if $R$ is isomorphic to $R_{1} \times R_{2}$, where $R_{1}$ is a semiring with multiplication of type $\left(T_{1}\right)$ and $R_{2}$ is a seniring with multiplication of type $\left(T_{2}\right)$.

We shall need the following lemma, whose proof is contained in $[1, p .75]$, to prove Theorem 1 .

LEMMA. Let $R$ be an anti-commutative semiring, then for arbitrary $x, y, z, \epsilon R$ we have

$$
\begin{aligned}
& \text { (i) } x^{2}=x \\
& \text { (ii) } x y z=x z
\end{aligned}
$$

PROOF OF THEOREM 1. Since $R$ is non empty, let $a \in R$. Set $R_{1}=R a$ and $R_{2}=a R$. By using the lemma, it is obvious that $R$ a and aR are semirings and multiplication in Ra is of type $\left(T_{1}\right)$ and multiplication in aR is of type $\left(T_{2}\right)$.

Let $f: R \rightarrow R a \times a R$, such that for each $x \in R$. $f(x)=(x a, a x)$.

then for $y \in R, f(y)=(y a, a y)$.

$$
\begin{aligned}
f(x+y) & =((x+y) a, a(x+y))=(x a+y a, a x+a y) \\
& =(x a, a x)+(y a, a y) \\
& =f(x)+f(y) . \\
f(x y) & =(x y a, a x y) \\
& =(x a y a, a x a y) \quad[\text { By part (11) of the Lemma] } \\
& =f(x) f(y) .
\end{aligned}
$$

Thus, $f$ is a homomorphism. 
To show $f$ is an isomorphism, let us define $g: R a x a R \rightarrow R$, such that $g(x a, a y)=x y$.

Then

$$
(g \circ f)(x)=g(f(x))=g(x a, a x)=x a^{2} x=x^{2}=x .
$$

and

$$
(f \circ g)(x a, a y)=f[g(x a, a y)]=f(x y)=(x y a, a x y)=(x a, a y) .
$$

This shows that $f$ is an isomorphism.

The proof for the converse is left to the reader.

THEOREM 2. Let $R$ be an anti-commutative semiring. Then for an arbitrary $x \in R, x+x=x$.

PROOF: As in the proof of Theorem 1, we have $\mathbf{x}=\mathbf{g}(\mathbf{x a}, \mathbf{a x})$.

Thus .

$$
\begin{aligned}
x+x & =g(x a+x a, a x+a x) \\
& =g\left(x^{2} a+x^{2} a, a x^{2}+a x^{2}\right) \\
& =g(x(x+x) a, a(x+x) x) \\
& =g(x a, a x) \\
& =x
\end{aligned}
$$

\section{REFERENCES}

1. LJAPIN, E.S. Semigroups, American Math Society Translation Providence, Rhode Is land (1963) 


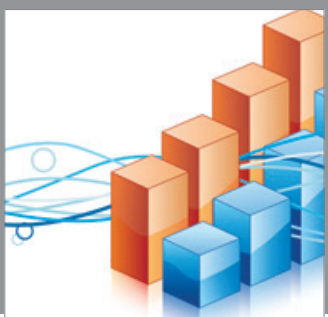

Advances in

Operations Research

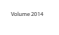

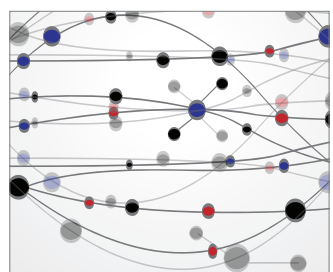

\section{The Scientific} World Journal
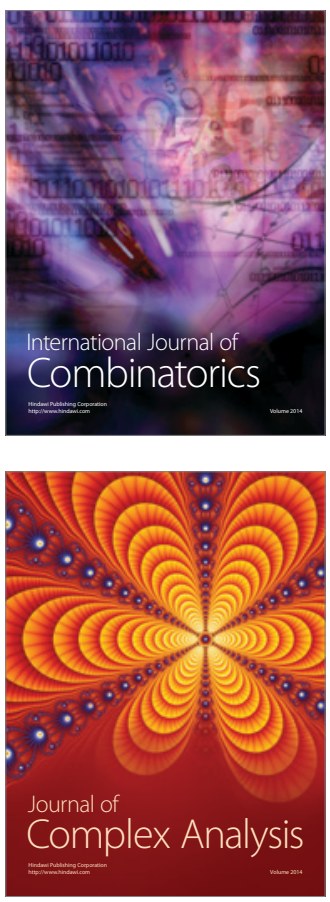

International Journal of

Mathematics and

Mathematical

Sciences
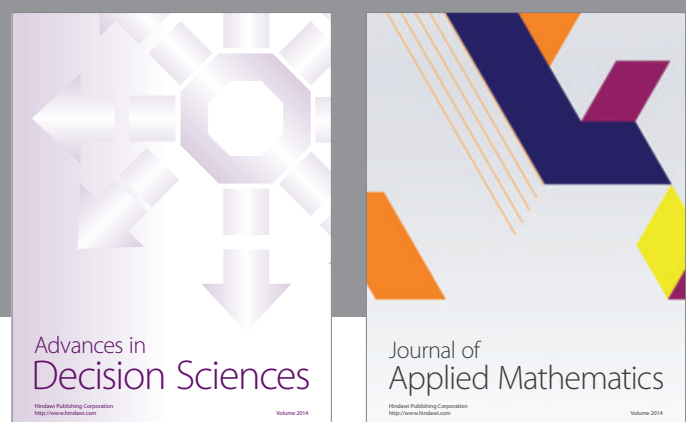

Journal of

Applied Mathematics
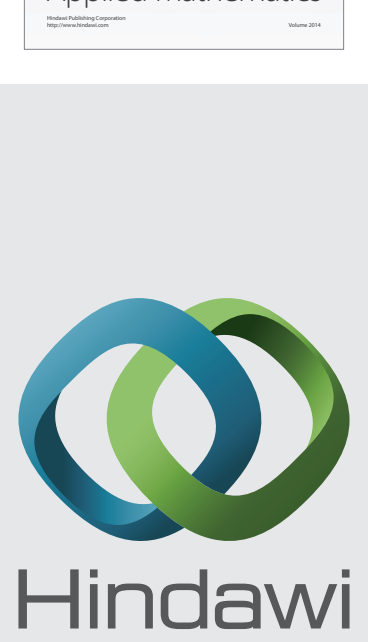

Submit your manuscripts at http://www.hindawi.com
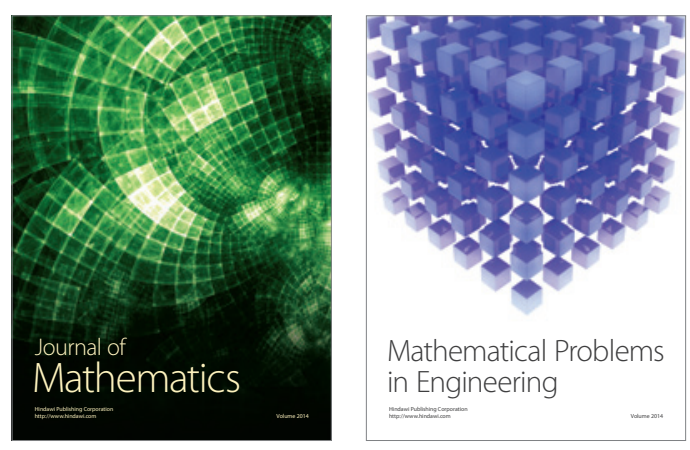

Mathematical Problems in Engineering
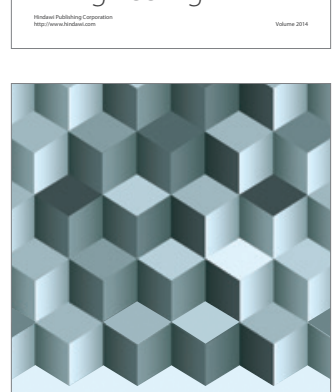

Journal of

Function Spaces
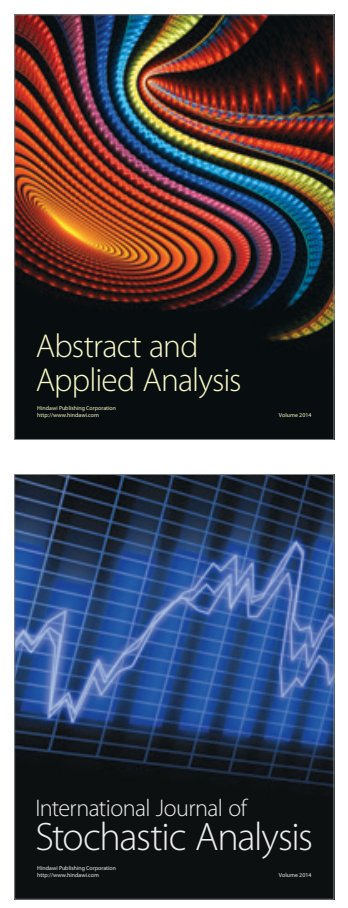

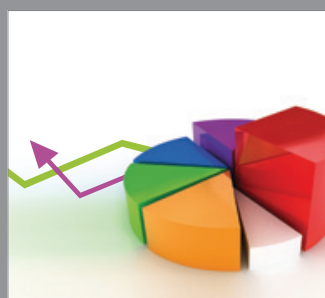

ournal of

Probability and Statistics

Promensencen
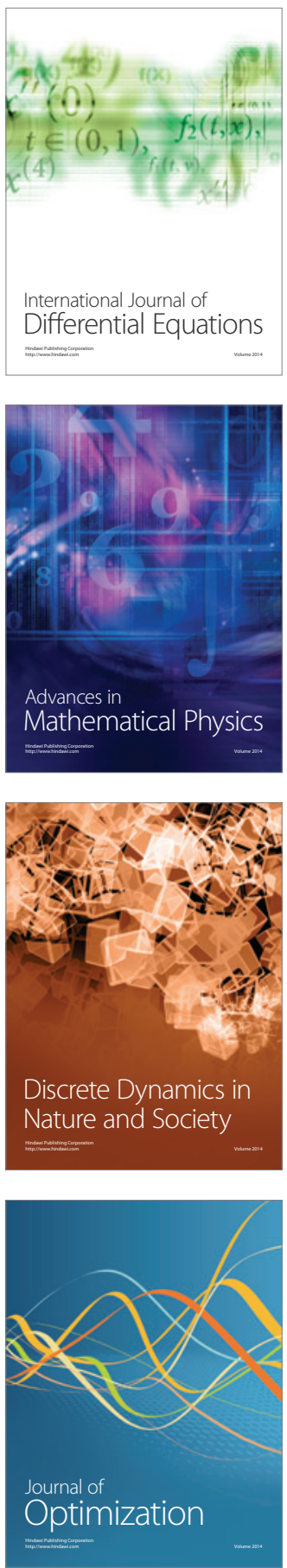